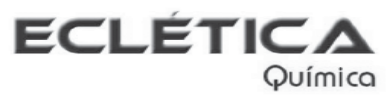

www.scielo.br/eq

Volume 29, número 2, 2004

\title{
Estudo da aplicação do processo foto-Fenton solar na degradação de efluentes de indústria de tintas
}

\author{
M. R. A. da Silva*, M. C. de Oliveira*, R. F. P. Nogueira* \\ * Instituto de Química - UNESP - Araraquara - SP.
}

\begin{abstract}
Resumo: Neste trabalho estudou-se a aplicabilidade do processo foto-Fenton utilizando irradiação solar no tratamento de dois efluentes (A e B) de uma indústria de tintas. Foi avaliada a influência da concentração inicial de peróxido de hidrogênio e ferro na remoção de carbono orgânico dissolvido (COD). Nos primeiros minutos de irradiação foi observada a descoloração e a remoção de odores. Para o efluente A, atingiu-se 75 \% de remoção de COD durante 60 minutos de irradiação. Para o efluente B, adições múltiplas de peróxido de hidrogênio foram necessárias para aumentar a remoção de COD, 43 \% em 60 minutos de irradiação. Os resultados obtidos mostram que o processo foto-Fenton solar pode ser aplicado ao tratamento dos efluentes gerados na indústria de tintas.
\end{abstract}

Palavras-chave: foto-Fenton; irradiação solar; tintas; fotodegradação; tratamento de efluentes.

\section{Introdução}

As tintas têm sua origem em tempos préhistóricos. Entretanto, somente nas últimas décadas a indústria de tintas conseguiu avanços atribuídos à pesquisa científica e à aplicação da engenharia moderna $\left[{ }^{1}\right]$. Este grande crescimento industrial trouxe também aumento no volume de resíduos gerados, os quais quando não corretamente dispostos ou tratados, podem causar sérios problemas de contaminação ambiental. Em todo o mundo há um esforço por parte das indústrias no sentido de diminuir a agressão ambiental causada por seus produtos $\left.{ }^{2}\right]$. O impacto ambiental causado pelas indústrias de tintas é de difícil avaliação, pois os efluentes têm uma composição variada devido à grande quantidade de matéria prima, reagentes e métodos de produção. Nestes efluentes podem-se encontrar sais, corantes, pigmentos, metais e outros compostos orgânicos de estruturas variadas, que são provenientes de etapas distintas do processo global.
O problema ambiental relacionado às tintas inicia-se desde a manufatura de seus componentes principais que são corantes ou pigmentos. Estudos recentes demonstraram que anualmente $12 \%$ dos corantes sintéticos são perdidos nos processos de produção e utilização, atingindo o ambiente sendo que sua coloração pode suprimir os processos fotossintéticos nos cursos d'águas [ $\left.{ }^{3}\right]$. Além do problema ambiental relacionado à cor, muitos dos produtos primários de degradação destes efluentes podem ser tóxicos e/ou não biodegradáveis [ $\left.{ }^{4}\right]$.

A complexidade dos efluentes das indústrias de tintas muitas vezes não permite que os mesmos sejam tratados por métodos convencionais. Algumas alternativas já têm sido propostas para minimizar o impacto causado por estas indústrias, como por exemplo, a utilização de água como solvente diminuindo a emissão de compostos orgânicos voláteis (VOC), desenvolvimento de sistemas de co-processamento de resíduos em fornos de cimento [5] e também 
um maior rigor no descarte e armazenamento de efluentes.

Diante disso, a busca por novas tecnologias aplicáveis ao tratamento de efluentes, assim como o desenvolvimento de processos limpos, com menor geração de resíduos, têm sido alguns dos grandes objetivos de pesquisadores preocupados com o ambiente. Os Processos Oxidativos Avançados (POA) têm se apresentado como uma alternativa eficiente para o tratamento de efluentes utilizando reações de oxidação iniciadas por radicais hidroxila (HO). Esses processos têm como principal vantagem a completa destruição de contaminantes orgânicos, convertendo-os em dióxido de carbono, água e sais inorgânicos $\left[^{6}\right]$, e vêm sendo muito estudados e empregados na remoção de corantes $\left[3 ; ;^{7} ;{ }^{8} ;{ }^{9}\right]$ e no tratamento de efluentes de indústrias têxteis $\left[{ }^{10},{ }^{11}\right.$, $\left.{ }^{12},{ }^{13},{ }^{14}\right]$.

Dentre os POA o processo de Fenton $\left[{ }^{15}\right]$ se destaca por ser uma poderosa fonte de radicais hidroxila a partir da mistura de sais de ferro e peróxido de hidrogênio:

$$
\mathrm{Fe}^{2+}+\mathrm{H}_{2} \mathrm{O}_{2} \rightarrow \mathrm{Fe}^{3+}+\mathrm{OH}^{-}+\mathrm{OH}
$$

Devido à alta reatividade do radical hidroxila, este pode iniciar diferentes tipos de reações com diferentes grupos funcionais formando radicais orgânicos instáveis que são posteriormente oxidados até $\mathrm{CO}_{2}, \mathrm{H}_{2} \mathrm{O}$ e ácidos inorgânicos provenientes do heteroátomo presente. A eficiência desta reação pode ser amplamente aumentada, quando se incide luz, o que é atribuído principalmente à fotorredução de Fe(III) a Fe (II), o qual reage posteriormente $\mathrm{com}_{2} \mathrm{O}_{2}$, ao aumento da decomposição de $\mathrm{H}_{2} \mathrm{O}_{2}$ pela absorção da luz UV e à fotólise de complexos orgânicos de Fe(III) gerados durante a decomposição. Além de alta eficiência, outras vantagens com relação a outros processos envolvem a pronta disponibilidade comercial do oxidante e baixo investimento capital

Tabela 1- Características dos efluentes “A” e "B”

\begin{tabular}{|c|c|c|c|c|c|}
\hline EFLUENTE & ODOR & COLORAÇÃo & $\begin{array}{c}\text { SOLUBILIDADE EM } \\
\text { ÁGUA }\end{array}$ & pH & $\begin{array}{c}\text { COT inicial } \\
\text { (ppm) }\end{array}$ \\
\hline A & Irritante & Preta & Totalmente solúvel & 4,75 & 662,3 \\
\hline B & $\begin{array}{c}\text { Odor } \\
\text { característico de } \\
\text { tinta }\end{array}$ & Azul & $\begin{array}{c}\text { Formação de uma } \\
\text { suspensão }\end{array}$ & 7,91 & 21786 \\
\hline
\end{tabular}

quando comparado aos processos de remediação disponíveis no mercado.

A ausência de estudos da utilização do processo foto-Fenton no tratamento de efluentes de indústrias de tintas, aliada à necessidade de diminuição do impacto ambiental causado por estes efluentes motivaram o presente trabalho. Foram feitos estudos de fotodegradação de 2 efluentes de indústria de tintas utilizando o processo foto-Fenton e luz solar como fonte de energia, visando a remoção de carbono orgânico.

\section{Material e Métodos Reagentes}

Todas as soluções foram preparadas utilizando água destilada. A concentração e os reagentes utilizados nos experimentos foram 0,5 mmol L-1 $\mathrm{Fe}\left(\mathrm{NO}_{3}\right)_{3} .9 \mathrm{H}_{2} \mathrm{O}$ (Mallinckrodt), $\mathrm{H}_{2} \mathrm{O}_{2}$ $30 \%$ (Synth), $9 \mathrm{~mol} \mathrm{~L}^{-1} \mathrm{H}_{2} \mathrm{SO}_{4}$ (Mallinckrodt), 0,06 mol L-1 metavanadato de amônio (Vetec) em 0,36 mol L-1 $\mathrm{H}_{2} \mathrm{SO}_{4}$ (Mallinckrodt).

\section{Amostras}

Foram feitos experimentos de fotodegradação em dois efluentes gerados por uma indústria de tintas com atividades voltadas para a linha de pigmentos, tintas serigráfica, acrílica e flexográfica. Os efluentes foram denominados pela indústria como "A" e "B", ambos com data de coleta em 20/06/2002 e a empresa não disponibilizou as composições. As características 
dos efluentes determinadas na data de recebimento estão descritas na tabela 1 .

\section{Processo de fotodegradação}

Os experimentos foram realizados em bateladas expondo as soluções contendo o efluente, sem tratamento prévio, e os reagentes ao sol utilizando um reator aberto de vidro de $3,5 \mathrm{~cm}$ de profundidade e $9 \mathrm{~cm}$ de diâmetro anteriormente estudado por Nogueira e colaboradores [ $\left.{ }^{16}\right]$. A solução foi mantida sob agitação magnética durante todo o tempo de exposição ao sol. Neste reator foram colocados $150 \mathrm{~mL}$ de efluente e o pH da solução foi ajustado para 2,5 pela adição de uma solução $9 \mathrm{~mol} \mathrm{~L}^{-1} \mathrm{H}_{2} \mathrm{SO}_{4}$, pH ótimo de acordo com trabalho anterior [ $\left.{ }^{17}\right]$. Adicionou-se o volume pré-estabelecido de uma solução $29 \%$ de peróxido de hidrogênio e expôs-se a solução diretamente à irradiação solar.

Durante os experimentos a intensidade da radiação solar foi monitorada a cada 1 minuto usando um radiômetro PMA 2100 Solar Ligth CO, na faixa de comprimento de onda entre 320 e 400 $\mathrm{nm}$, com o sensor posicionado horizontalmente. Para cada experimento foi calculada a média da intensidade luminosa para o período de irradiação e também, para efeito de comparação dos resultados $\left[{ }^{18}\right]$, medida a dose de energia acumulada no período, que é a integração da intensidade de energia irradiada em função do tempo.

\section{Análises Químicas}

Devido à formação de um precipitado durante a fotodegradação, as amostras foram centrifugadas e alíquotas do sobrenadante foram diluídas 32 e 301 vezes para os efluentes A e B, respectivamente. Nestas amostras foram feitas determinações de carbono orgânico dissolvido (COD).

Para caracterização dos efluentes na data do recebimento foi feita a determinação de Carbono Orgânico Total (COT). Para efeito de comparação com as amostras fotodegradadas, estes efluentes também foram centrifugados e não foram observadas variações entre COD e COT.

Ambas as determinações foram feitas em um analisador de carbono TOC 5000A Shimadzu. A eficiência de degradação foi avaliada pelo decaimento da concentração relativa de carbono orgânico dissolvido $\left(\mathrm{COD} / \mathrm{COD}_{0}\right)$ do efluente em função do tempo de irradiação.

O peróxido de hidrogênio residual é um parâmetro importante no processo de fotodegradação, visto que uma vez consumido, a reação de fotodegradação torna-se mais lenta ou não prossegue. A concentração de peróxido de hidrogênio foi monitorada espectrofotometricamente pela geração de peroxovanádio com máximo de absorção em 446 nm [ $\left.{ }^{19}\right]$, formado pela reação de peróxido de hidrogênio com metavanadato de amônio:

$\mathrm{VO}_{3}{ }^{-}+4 \mathrm{H}^{+}+\mathrm{H}_{2} \mathrm{O}_{2} \rightarrow \mathrm{VO}_{2}{ }^{3+}+3 \mathrm{H}_{2} \mathrm{O}$

Para análise, foram adicionados 4,00 $\mathrm{mL}$ de amostra a 1,60 mL de metavanadato de amônio e completado o volume com água deionizada para $10,0 \mathrm{~mL}$ e a absorbância medida em espectrofotômetro UV-Mini-Shimadzu 1240.

\section{Resultados e Discussão}

Durante os primeiros minutos de irradiação dos efluentes A e B foi possível observar a formação de precipitados, que foram facilmente separados por centrifugação durante aproximadamente 5 minutos. Foi obtida uma solução transparente e um sólido preto e azul para os efluentes A e B, respectivamente. A composição destes precipitados não foi alvo de estudo. Para o efluente não tratado esta separação não foi obtida mesmo após um tempo de 45 minutos de centrifugação. A separação destas fases na fotodegradação dos efluentes A e B possibilita uma facilidade no tratamento, visto que após a separação pode-se tratar o líquido independentemente e o resíduo sólido ter um tratamento mais adequado, ou eventualmente um reaproveitamento no processo de produção. A separação de fases também propicia uma descoloração do efluente, o que é importante considerando todos os problemas associados à cor do efluente.

Também nos primeiros minutos de irradiação, foi observada a eliminação dos fortes odores característicos de cada efluente. Sugerindo que um curto tempo de irradiação permite a degradação imediata de compostos de forte odor. 
Fotodegradação do efluente A

Para o efluente A foram feitos experimentos nos quais variou-se apenas a quantidade de energia solar recebida durante o experimento. Nestes experimentos de fotodegradação não foi necessária a reposição de peróxido, pois as análises residuais demonstravam haver um excesso do reagente. Na figura 1 é mostrado o decaimento da concentração relativa de COD $\left(\mathrm{COD} / \mathrm{COD}_{0}\right)$ do efluente A durante irradiação sob diferentes doses de energia. No experimento 1 , com menor dose de energia, observou-se que a remoção de COD foi de aproximadamente $54 \%$ após 60 minutos de irradiação. Já para o experimento 2, com maior intensidade luminosa, em 60 minutos de irradiação obteve-se aproximadamente $76 \%$ de remoção de COD (Figura 1).

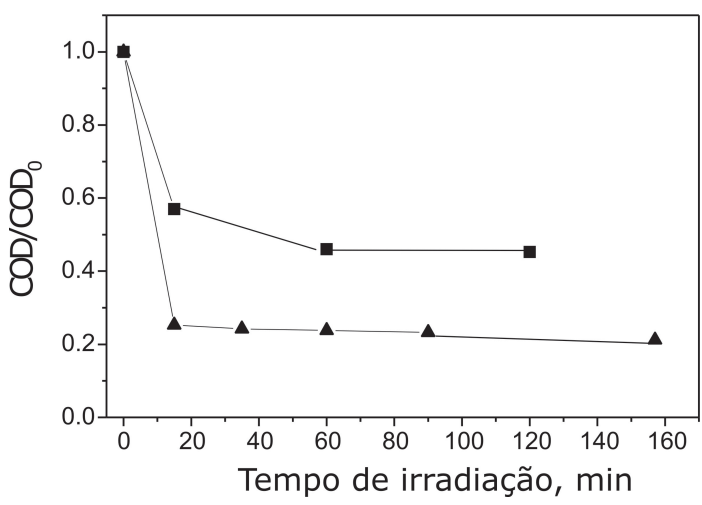

Figura 1 - Remoção de COD na fotodegradação do efluente A. $\left[\mathrm{Fe}^{3+}\right]=0,80 \mathrm{mmol} \mathrm{L}^{-1},\left[\mathrm{H}_{2} \mathrm{O}_{2}\right]$ inicial $=0,30 \mathrm{~mol} \mathrm{~L}^{-1}$. Dose de energia: experimento $1(120$ minutos $)=16,35 \mathrm{~J} \mathrm{~cm}^{-2} \mathrm{e}$ experimento 2 (90 minutos) $=16,69 \mathrm{~J} \mathrm{~cm}^{-2}$.

Observando os resultados dos experimentos 1 e 2 mostrados na figura 1, podese verificar que este último apresenta maior eficiência de remoção de COD, resultante da maior dose de energia solar no experimento 2. Observase também que após uma grande diminuição da concentração relativa de COD, nos primeiros 15 minutos de irradiação, esta permanece constante mesmo após 160 minutos de irradiação, o que indica que compostos recalcitrantes podem ter sido gerados durante o processo de fotodegradação.
Fotodegradação do efluente $B$

Para o efluente B pode-se verificar que a porcentagem de remoção de COD, utilizando 0,30 mol L ${ }^{-1}$ de $\mathrm{H}_{2} \mathrm{O}_{2}$ e $1,60 \mathrm{mmol} \mathrm{L}^{-1} \mathrm{Fe}^{3+}$, foi de aproximadamente $23 \%$ após 60 minutos de irradiação e que esta se manteve praticamente constante mesmo após a reposição de peróxido de hidrogênio feita pela adição de $5 \mathrm{~mL}$ de peróxido de hidrogênio 29\% (0,05 mol) (figura 2). As análises de peróxido de hidrogênio residual revelaram que o efluente $B$ consumiu peróxido de hidrogênio muito rapidamente, pois após 35 minutos de irradiação este reagente já havia sido consumido totalmente, exigindo sua reposição durante os experimentos. Isto pode ser devido à presença de uma maior quantidade de ferro em relação ao efluente A. Desta forma, estudou-se a reposição de peróxido de hidrogênio em um tempo inferior a 60 minutos. Pode-se observar que antes da reposição de peróxido de hidrogênio feita em 40 minutos, a remoção de COD alcançava valores próximos a $21 \%$ em 35 minutos de irradiação e após a reposição de peróxido de hidrogênio essa remoção teve seu valor aumentado para 34\% após 60 minutos e ao final da irradiação, aos 157 minutos, obteve-se uma remoção próxima a 51\% (Figura 2).

Ainda para este efluente estudou-se a adição de uma menor concentração inicial de peróxido de hidrogênio seguida de adições em 30 e 50 minutos de $526 \mathrm{~mL}$ de peróxido de hidrogênio $29 \%$. Observou-se após 60 minutos uma remoção de aproximadamente $43 \%$ de COD (figura 2).

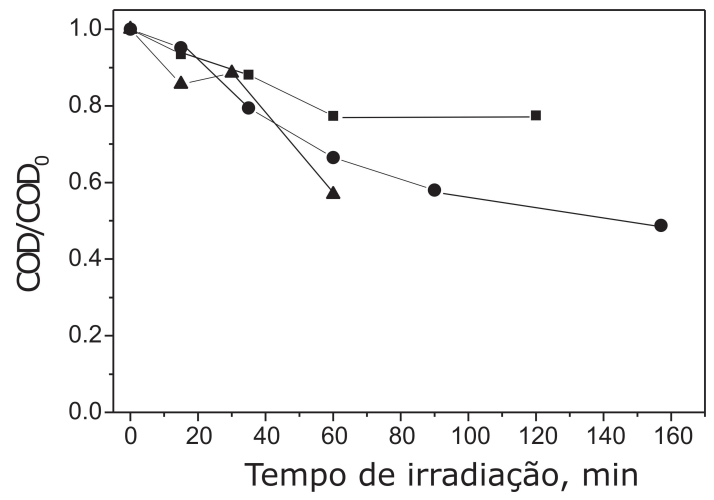

Figura 2 - Remoção de COD na fotodegradação do efluente B utilizando luz solar. $\left[\mathrm{Fe}^{3+}\right]$ 1,60 $\mathrm{mmolL}^{-1}$ 
Estes resultados levam a concluir que a adição inicial de uma menor concentração de peróxido de hidrogênio $\left(20 \mathrm{mmol} \mathrm{L}^{-1}\right)$ seguida de adições múltiplas (30 e 50 minutos) do mesmo é o procedimento mais eficiente, já que após 60 minutos de irradiação 43\% de remoção de COD foram atingidos, enquanto que comparando com utilização de 0,30 mol L ${ }^{-1}$ de $\mathrm{H}_{2} \mathrm{O}_{2}$ e reposição única em 40 e 60 minutos foram obtidos 33,5 e 22,6 \%, respectivamente.

Os resultados mostrados para os dois efluentes, nas figuras 1 e 2, indicam que os produtos gerados após 40 minutos de fotodegradação apresentam uma velocidade de degradação mais baixa que os compostos iniciais. Isto é evidenciado no efluente $B$, no qual a reposição de peróxido de hidrogênio aos 40 minutos não provocou uma diminuição acentuada de COD, pois no momento da reposição a remoção de COD era de $34 \%$ e passados 50 minutos de reposição ocorreu um aumento de somente $8 \%$ na remoção de COD. Outra prova de que a velocidade de degradação dos produtos é lenta se deve ao fato de a reação não parar mesmo após cessar a irradiação. Na tabela 2 são mostrados resultados de determinações de COD de amostras armazenadas no escuro após a fotodegradação. Os resultados indicam a potencialidade do processo ser aplicado também sem irradiação. Esta menor velocidade de degradação dos produtos formados após 40 minutos de irradiação deve-se provavelmente à geração de intermediários menos susceptíveis à degradação quando comparado ao composto inicial, sendo que a identificação destes

Tabela 2: Porcentagem de remoção de COD para amostras armazenadas no escuro após a irradiação solar.

\begin{tabular}{|c|c|c|c|c|}
\hline \multirow{2}{*}{} & \multicolumn{4}{|c|}{$\%$ Remoção de COD } \\
\cline { 2 - 5 } & \multicolumn{2}{|c|}{ Efluente A } & \multicolumn{2}{c|}{ Efluente B } \\
\hline $\begin{array}{c}\text { Tempo de } \\
\text { armazenagem (h) }\end{array}$ & $\begin{array}{c}\text { Menor intensidade } \\
\text { luminosa }\end{array}$ & $\begin{array}{c}\text { Maior intensidade } \\
\text { luminosa }\end{array}$ & $\begin{array}{c}\text { Reposição de } \\
\mathrm{H}_{2} \mathrm{O}_{2} \text { em } 60 \\
\text { minutos }\end{array}$ & $\begin{array}{c}\text { Reposição de } \\
\mathrm{H}_{2} \mathrm{O}_{2} \text { em } 40 \\
\text { minutos }\end{array}$ \\
\hline 0 & 54,0 & 76,2 & 15,7 & 33,5 \\
\hline 180 & 94,3 & ----- & 48,7 & ----- \\
\hline 240 & ------- & 82,1 & ------ & 57,1 \\
\hline
\end{tabular}

não estava entre os objetivos deste trabalho. No entanto é importante salientar que a reação continua ocorrendo rumo à mineralização, o que foi evidenciado nos resultados de remoção de COD das amostras armazenadas no escuro.

Comparando-se a eficiência de fotodegradação dos efluentes A e B verificou-se que o efluente $A$ apresentou valores de porcentagem de remoção de COD mais altos que o efluente $B$, o que evidencia sua maior facilidade de tratamento pelo processo foto-Fenton com irradiação solar. Isto decorre principalmente de sua menor concentração de COD cerca de 30 vezes menor do que a do efluente $\mathrm{B}$. As análises de peróxido de hidrogênio residual mostram que a quantidade de peróxido de hidrogênio inicial foi excessiva para os dois efluentes podendo este valor ser diminuído, reduzindo os custos do processo. Uma possível causa da diminuição na porcentagem de fotodegradação em altas concentrações de peróxido de hidrogênio é o consumo do radical hidroxila em presença de alta concentração de peróxido de hidrogênio formando um novo radical, o hidroperoxil, com menor poder de oxidação, como mostra a equação a seguir [ ${ }^{2020}$ K. A. Hislop, J. R. Bolton, Environ. Sci. Technol. 33 (1999) 3119.].

$\mathrm{OH}^{\cdot}+\mathrm{H}_{2} \mathrm{O}_{2}{ }^{\circledR} \mathrm{H}_{2} \mathrm{O}+\mathrm{HO}_{2}$.

\section{Conclusão}

Os resultados obtidos neste estudo mostram que o processo de tratamento de efluentes de indústrias de tintas por processo foto-Fenton 
utilizando luz solar como fonte de irradiação é eficiente para remoção de COD, odor e cor dos efluentes tratados em um curto período de tempo. O processo Fenton ocorrido no escuro, após os períodos de irradiação, deu prosseguimento ao processo de mineralização dos compostos presentes em ambos os efluentes. $\mathrm{O}$ baixo custo deste processo, aliado à química limpa (não utilização de reagentes tóxicos) e à sua alta eficiência, o torna viável para aplicação no tratamento de efluentes de indústrias de tintas. Maiores estudos serão necessários para esta aplicação, como por exemplo, a identificação de produtos de degradação, a otimização do sistema para utilização em escala industrial e avaliação da biodegradabilidade do efluente após o tratamento com processo foto-Fenton para combinação a um processo biológico.

\section{Agradecimentos}

M.R.A. Silva e M.C. Oliveira agradecem à FAPESP e ao CNPq pelas bolsas concedidas.

Recebido em 03/02/04 Aceito em 07/04/04

M. R. A. da Silva, M. C. de Oliveira, R. F. P. Nogueira. Photo- Fenton applicability in the treatment of ink wastewater industry.

Abstract: The photo- Fenton process was evaluated for the treatment of two effluents (A and B) from ink industry. The influence of hydrogen peroxide and iron (II) concentration on the dissolved organic carbon (DOC) removal was evaluated. In the first minutes of irradiation the color and odors were removed. For the effluent A 75\% of DOC removal was reached in 60 min irradiation. For the effluent $\mathrm{B}$, multiple additions of $\mathrm{H}_{2} \mathrm{O}_{2}$ were necessary to improve the COD removal, reaching $43 \%$ of TOC in $60 \mathrm{~min}$ irradiation. The results obtained demonstrate that the solar photo-Fenton process can be applied for the treatment of effluents generated by ink industry.

Keywords: photo-Fenton, solar irradiation, ink, photodegradation, wastewater treatment .

\section{Referências}

[1] R. N. Shreve, J. Jr. Brink, A. Industria de Processos Químicos, Editora Guanabara Koogan S.A., Rio de Janeiro, 4 ed., 1977, cap. 24.

[2] O Esforço para produzir tintas que não agridam o meio ambiente. As Tintas. Disponível em: http:/ /www.mundocor.com.br/tinta_meioambiente.htm. Acesso em 30 de outubro de 2002.

[3] I. Arslan, A. I. Balcioglu, D. W. Bahneman, Dyes Pigm. 47 (2000) 207.

[4] H. D. Mansilla, C. Lizama, A. Gutarra, J. Rodríguez, In: BLESSA, M. A. Eliminación de contaminantes por fotocatálisis heterogénea. Argentina: CYTED, (2001) cap. 3.

[5] Cavo: gestão de resíduos ampliada. Saneamento Ambiental 65 (2000) 46.

[6] O. Legrini, E. Oliveros, A. M. Braun, Chem. Rev. 93 (1993) 671.

[7] J. Bandara, C. Morrison, J. Kiwi, C. Pulgarin, P. Peringer, J. Photochem. Photobiol., A 99 (1996) 57.
[8] R. Aplin, A. J. Feitz, T. D. Waite, Wat. Sci. Technol 44 (2001) 23.

[9] H. Park, W. Chu, J. Photochem. Photobiol., A 159 (2003) 241.

[10] S. H. Lin, C. C. Lo, Water Res. 31 (1997) 2050.

[11] S. F. Kang, C. H. Liao, S. T. Po, Chemosphere 41 (2000) 1287.

[12] M. Perez, F. Torrades, X. Doménech, J. Peral, Water Res. 36 (2002) 2701.

[13] M. Rodriguez, V. Sarria, S. Esplugas, C. Pulgarin J. Photochem. Photobiol., A 151 (2002) 129.

[14] M. Neamtu, A. Yediler, I. Siminiceanu, A. Kettrup, J. Photochem. Photobiol., A 161(2003) 87.

[15] C. Walling, Acc. Chem. Res.6 (1975) 125.

[16] R. F. P. Nogueira, A. G. Trovo, D. F. Modé, Chemosphere, 48 (2002) 385. 
[17] R. F. P. Nogueira, J. R. Guimarães, Water Res. 34 (2000) 895.

[18] R. F. P. Nogueira, A. G. Trovo, Paterlini, W.

C. Wat. Sci. Technol, 49 (2004) no prelo.
[19] M. C. Oliveira, R. F. P. Nogueira, J. A. G. Neto, W. F. Jardim, J. J. R. Rohwedder, Quím. Nova 24 (2001) 188.

[20] K. A. Hislop, J. R. Bolton, Environ. Sci. Technol. 33 (1999) 3119. 